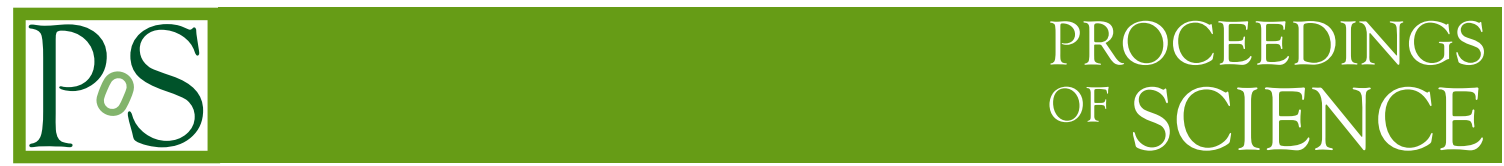

\title{
ATLAS Standard Model Results
}

\author{
Pavel Jež ${ }^{* \dagger}$ \\ Niels Bohr Institute, University of Copenhagen \\ E-mail: jez@nbi.dk
}

The Standard Model results obtained in $\sqrt{s}=7 \mathrm{TeV}$ proton-proton collisions at the Large Hadron Collider using the ATLAS detector are reviewed. The measurements presented in this contribution are using all data collected in 2010 and up to $1 \mathrm{fb}^{-1}$ of proton-proton collisions collected in 2011. The topics covered in this document are soft and hard QCD processes, the production of $\mathrm{W}$ and $\mathrm{Z}$ bosons and top quark cross-section and mass measurements.

The XXth International Workshop High Energy Physics and Quantum Field Theory September 24 - October 1, 2011

Sochi, Russia

*Speaker.

${ }^{\dagger}$ On behalf of the ATLAS Collaboration 


\section{Introduction}

The Standard Model currently provides the simplest and most accurate description of the processes observed in the particle physics experiments. The validation of the Standard Model predictions is therefore crucial for all HEP experiments. In the beginning, the abundant Standard Model processes, collectively known as soft QCD, help to calibrate the detector response to charged particles and to measure total inelastic $p p$ cross-section at the centre-of-mass energy $7 \mathrm{TeV}$. With the increasing amount of data more rare processes - like production of multiple jets with high transverse momentum, high $p_{\mathrm{T}}$ photon production, production of $\mathrm{W}$ and $\mathrm{Z}$ bosons or top quarks - are available to study at the new energy frontier of LHC.

Detailed study of such events helps to validate the predictions of QCD and Electroweak Standard Model beyond the leading order of the perturbation theory. The results of Standard Model measurements are also invaluable in the searches of the new physics: the Standard Model processes form major background in a bulk of those searches. Finally, the precise measurement of the Standard Model parameters can constrain the properties of possible additional particles that can appear in the loop corrections of the Standard Model diagrams.

The ATLAS detector [1] is one of the two general purpose detector at the Large Hadron Collider (LHC) [2] in CERN ${ }^{1}$. ATLAS consists of an inner tracking detector surrounded by a superconducting solenoid, electromagnetic and hadronic calorimeters and a muon spectrometer submerged in a toroidal magnetic field. The inner detector uses silicon pixels and strips as well as straw tube tracker to provide precision tracking up to $|\eta|<2.5^{2}$. The calorimeters in ATLAS are sampling calorimeters with either liquid argon or scintillating tiles as active media. They cover the pseudorapidity range up to $|\eta|<4$.9. In the region $|\eta|<2.5$, the electromagnetic calorimeter is finely segmented to provide precise input for electron and photon reconstruction and identification. The only detectable particles that can usually traverse the calorimeters without being stopped are muons. The muon spectrometer uses straw tubes in high-precision tracking chambers to measure muon momentum up to $|\eta|<2.7$.

The LHC started colliding protons at $7 \mathrm{TeV}$ centre-of-mass energy in 2010 and ATLAS detector managed to collect $45 \mathrm{pb}^{-1}$ of data that year. After the winter shut-down, the LHC was delivering beams of much higher intensity in 2011, resulting in $5.25 \mathrm{fb}^{-1}$ of data recorded in 2011. This contribution will show results obtained with the full 2010 dataset and the first $1 \mathrm{fb}^{-1}$ of 2011 data collected between February and July 2011. The high intensity of proton beams in 2011 also resulted in multiple $p p$ interactions every time the beams crossed, so-called pile-up. In 2011 data used for measurements presented in this contribution, the maximum mean number of interactions was around 8. In 2010, the maximum amount of pile-up were roughly 3 interactions per beam crossing.

\footnotetext{
${ }^{1}$ European Organization for Nuclear Research in Geneva, Switzerland - www.cern.ch

${ }^{2}$ ATLAS uses right-handed coordinate system with $y$ axis pointing vertically upwards and $x$ axis pointing towards the centre of the LHC ring. The azimuthal angle measured w.r.t. $x$ axis in $x y$ plane is denoted $\phi$, while $\theta$ is the angle from the $z$ axis. The pseudorapidity $\eta$ is defined as
}

$$
\eta=-\ln \tan \frac{\theta}{2}
$$




\section{Soft QCD processes}

The processes of interest at hadron colliders typically involve high transverse momentum transfer from initial to final state and production of particles with significant $p_{\mathrm{T}}$. However, most of the $p p$ collisions result in events with low $p_{\mathrm{T}}$ transfer and a few or no particles with significant $p_{\mathrm{T}}$ : soft $Q C D$. They form unavoidable background to all observables, but unfortunately are generally not well understood due to their non-perturbative nature. Various phenomenological models are used for the soft QCD description, but those models need to be tuned in order to describe data correctly.

ATLAS performed minimum-bias measurements using low pile-up ${ }^{3}$ data from the beginning of 2010 [3]. Minimum-bias (MB) events are selected using minimal event selection criteria and are dominated by purely soft QCD scattering. The MB events were recorded using MBTS trigger that required at least one count in either of the scintillation counters mounted in the forward and backward regions of the ATLAS detector. The MBTS covers region $2.09<|\eta|<3.84$.

The distributions of charged particles were measured in several phase-spaces. The most inclusive selection required just 2 charged particles with $p_{\mathrm{T}}>100 \mathrm{MeV}$. ATLAS measured the charged particle multiplicity as well as multiplicity distribution versus $\eta$ and $p_{\mathrm{T}}$. This was compared to the prediction of various Monte-Carlo (MC) models. The MC09 tune of Pythia 6 [4] was obtained using the data available before LHC delivered first $\sqrt{s}=7 \mathrm{TeV}$ collisions. Given the differences in predicted and observed values, new MC tuning was necessary. The tune AMBT1 uses data obtained from $7 \mathrm{TeV}$ collisions by selection of at least 6 charged particles with $p_{\mathrm{T}}>500 \mathrm{MeV}$ in order to suppress diffractive processes. Although AMBT1 fails to describe inclusive soft QCD events completely, it brings significant improvement over the pre-LHC tunes and describe well the non-diffractive MB events.

ATLAS provided also the first measurement of inelastic $p p$ cross-section at $\sqrt{s}=7 \mathrm{TeV}$ [5]. Events were triggered by requiring at least two counts in the MBTS. The mean pseudorapidity of the two particles separated by the largest pseudorapidity gap is used to assign all particles with greater $\eta$ to one system and all others to the other one. If we denote the system with larger invariant mass as $X$ and its mass as $M_{X}$, we can define $\xi=M_{X}^{2} / s$. MBTS cannot register events that produce only particles with $|\eta|>3.84$. This limits phase-space to $\xi>5 \times 10^{-6}$.

The measured cross-section was also extrapolated to the elastic limit $\xi>m_{p}^{2} / s$. Those results as well as phenomenological predictions are shown in fig. 1. The value measured for $\xi>5 \times 10^{-6}$ is $60.3 \pm 2.1 \mathrm{mb}$, that is somewhat lower then the theory predictions. However, the extrapolated value $\sigma_{\text {inel }}=69.4 \pm 2.4$ (exp.) \pm 6.9 (extr.) mb agree with most models.

\section{Hard QCD}

Most of the processes with large $p_{\mathrm{T}}$ transfer (hard processes) result in a production of jet(s). The jets are result of the hadronization of the high- $p_{\mathrm{T}}$ partons (quarks or gluons) produced in the hard scattering process: the hard parton radiates other partons that are predominantly collimated with the mother parton due to the nature of the strong QCD coupling. The colour carrying partons produced by this showering ultimately undergo combination to colourless hadrons that are observed

\footnotetext{
${ }^{3}$ The probability of having more than one interaction in the events selected for the MB study was $0.1 \%$.
} 


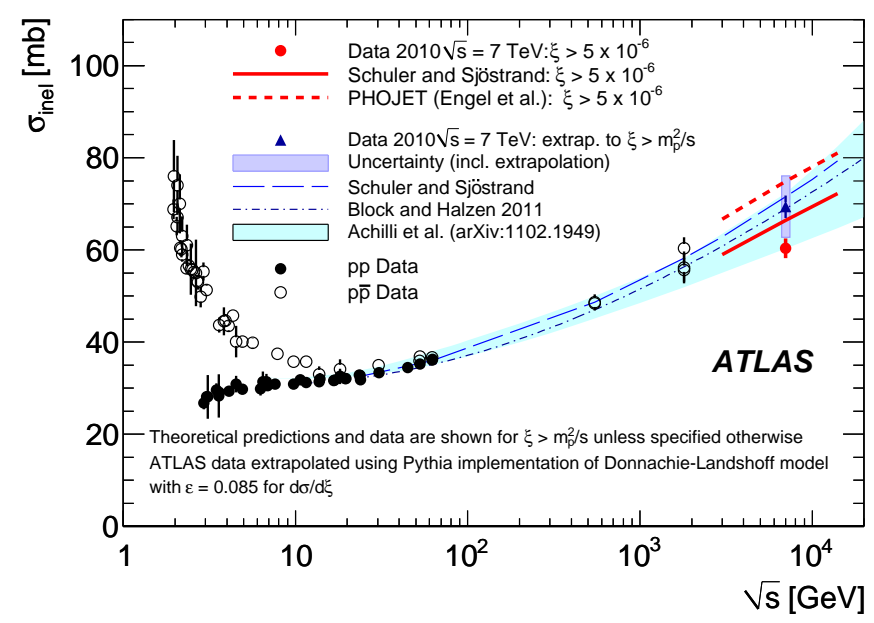

Figure 1: The inelastic $p p$ and $p \bar{p}$ cross-section versus centre-of-mass energy. The rightmost data points were provided by ATLAS, while others are from older experiments. Two different phenomenological models (red lines) are used as a comparison with measured cross-section for $\xi>5 \times 10^{-6}$ (red point), while three different models (blue lines and light blue area) are used for comparison with the ATLAS measurement extrapolated to elastic limit (dark blue triangle). The experimental uncertainty is indicated by the error bar, while the total uncertainty after extrapolation is represented by the blue shaded area. Figure is from [5].

in the detector. A shower of highly collimated hadrons is called jet and its reconstruction can help us to study the properties of the original quark or gluon. The hard QCD measurements can be compared with the available NLO perturbative QCD (pQCD) predictions, thus validating the theory in the new kinematic regime or providing possible hints of the new physics in case of disagreement between the measured values and $\mathrm{PQCD}$ predictions.

In ATLAS, jets are reconstructed from the energy deposits in the calorimeter cells [6]. Starting from the cells with deposits more than 4 times over the noise threshold, these cells are combined in an iterative way with other cells passing somewhat lower energy thresholds in order to form 3-dimensional clusters. Those clusters are merged using anti- $k_{t}$ algorithm [7] to create jets. The anti- $k_{t}$ algorithm is infrared and collinear safe and produces regular, cone-like jets. The values of distance parameter $R$ (corresponding to a typical jet cone radius) used in ATLAS are $R=0.4$ or $R=0.6$. The distance of 2 clusters $i$ and $j$ (as well as most of the objects in ATLAS) is defined using $(\phi, \eta)$ metric as $\Delta_{i j}=\sqrt{\left(\Delta \phi_{i j}\right)^{2}+\left(\Delta \eta_{i j}\right)^{2}}$.

The calorimeter response must be corrected in order to account for the fact that the ATLAS calorimeters are non-compensating, for the presence of inactive material and also for the out-ofcone effects. Baseline energy scale is so-called electromagnetic (EM) scale. It was derived using $Z \rightarrow e e$ events and test beam data. The EM+JES calibration [8] restores the hadronic jet energy scale (JES) using a data-derived offset pile-up subtraction and employing $(\eta, \mathrm{E})$ - dependent calibration constants derived from MC simulations. The EM+JES scheme is used to calibrate jets with $p_{\mathrm{T}}>20 \mathrm{GeV}$ and $|\eta|<4.5$. The JES uncertainty is dominant experimental uncertainty for numerous LHC analyses. Data-driven techniques were employed to reduce this uncertainty down to $2.5 \%$ in the central region $(|\eta|<0.8)$ for jets with $60 \mathrm{GeV}<p_{\mathrm{T}}<800 \mathrm{GeV}$. The JES uncertainty is largest for low- $p_{\mathrm{T}}$ very forward jets $\left(20 \mathrm{GeV}<p_{\mathrm{T}}<30 \mathrm{GeV}\right.$ and $\left.3.2 \leq|\eta|<4.5\right)$ where it 


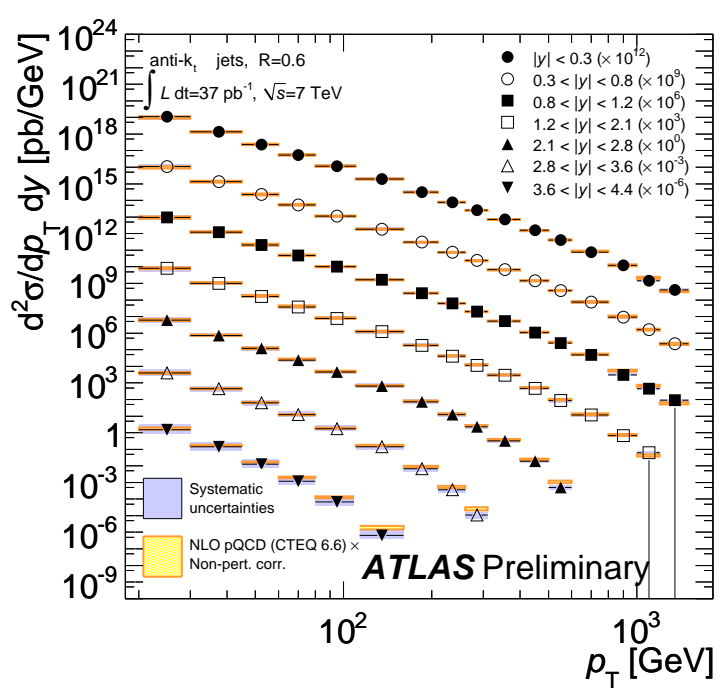

Figure 2: Inclusive jet double-differential cross-section as a function of jet $p_{\mathrm{T}}$ in various rapidity intervals. The jets were identified using anti- $k_{t}$ algorithm with $R=0.6$. For convenience, the cross-sections are multiplied by factors indicated in the legend. The data are compared to NLO pQCD predictions. Figure is from [9].

amounts to $14 \%$.

The inclusive jet cross-section has been measured using 2010 data in 7 rapidity intervals for jets with $20 \mathrm{GeV}<p_{\mathrm{T}}<1.5 \mathrm{TeV}$ [9]. The resulting values span over 12 orders of magnitude and are presented in fig. 2. The experimental uncertainty is dominated by JES, especially for large rapidity intervals. The events for this analysis were selected by a single jet trigger. Apart from JES, all other corrections for detector inefficiencies and resolution are performed in a single step using a bin-by-bin correction derived from MC simulation.

Besides JES, the other sources of experimental uncertainty were jet energy and angular resolution, the efficiency of jet trigger and jet reconstruction and finally the spectral shape modeling in the MC. Fig. 3 shows comparison of measured values of jet cross-section to NLO pQCD. The fixedorder NLO calculations of parton-level cross-sections are provided by NLOJet++ program [10] along with CTEQ 6.6 [11] NLO parton distribution functions (PDFs). The non-perturbative corrections (parton shower and hadronization) are done by Pythia [4]. The uncertainties on the theory predictions are dominated by the PDF uncertainties. Fig. 3 shows some differences at high jet $p_{\mathrm{T}}$ and $|y|$, but in general data are in agreement with NLO predictions within uncertainties.

Another analysis is checking the dijet azimuthal decorrelation [12]. This measurement provides a very interesting method to access multi parton production using only the two jets with the highest $p_{\mathrm{T}}$ in the event. In case of a pure dijet events, the two jets tend to be back-to-back in the transverse plane due to momentum conservation: $\Delta \phi=\pi$ and azimuthal decorrelation is small. In events with several high- $p_{\mathrm{T}}$ jets the azimuthal angle tend to be significantly smaller than $\pi$. QCD also predicts that the shape of $\Delta \phi$ distribution becomes more narrow with increase of the leading jet $p_{\mathrm{T}}$. A detailed understanding of events with large azimuthal decorrelations is important in searches for new phenomena with dijet signatures. The measured values of $\Delta \phi$ distributions agree well with 

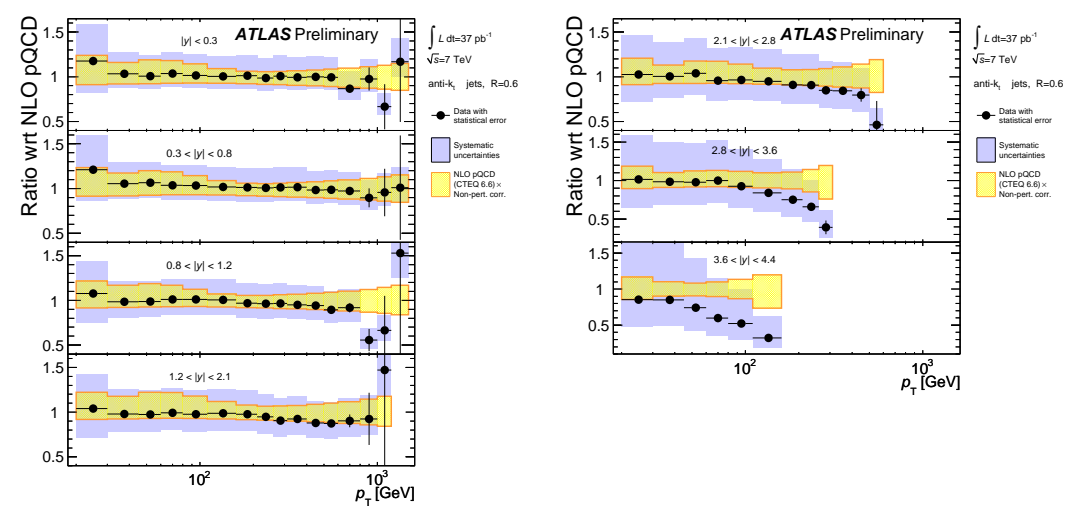

Figure 3: Ratio of measured inclusive jet double-differential cross-section and its NLO Standard Model expectation as a function of jet $p_{\mathrm{T}}$ in various rapidity intervals. The jets were identified using anti- $k_{t}$ algorithm with $R=0.6$. The ratio of the data to NLO pQCD predictions is shown and the total experiment and theoretical uncertainties indicated. Figures are from [9].

LO and NLO predictions (provided by NLOJet++ with fastNLO) within the uncertainties.

The production of dijets with a veto on central jet activity was studied as well [13]. In this measurement a jet veto much larger than $\Lambda_{\mathrm{QCD}}$ is used to identify absence of additional activity in order to study diverse range of pQCD phenomena (e.g. BKFL-like dynamics [14], that are expected to be important for large rapidity intervals, or wide-angle soft-gluon radiation). In case of widely separated jets and jet veto scale small compared to their $p_{\mathrm{T}}$, it is possible to study also the colour singlet exchange. The measurement therefore brings some information about phase-space regions that may be not described adequately by the standard event generators.

The jets in this analysis are reconstructed using anti- $k_{t}$ algorithm with $R=0.6$ and are required to have $p_{\mathrm{T}}>20 \mathrm{GeV}$ and $|y|<4.4$. The dijet system is defined as either two jets with largest $p_{\mathrm{T}}$ (to study wide-angle soft gluon radiation) or the most forward and the most backward jet (to study BKFL-like dynamics). In both definitions, the mean $p_{\mathrm{T}}$ of the dijet system is required to be greater than $50 \mathrm{GeV}$. The event is vetoed if there is a jet in the rapidity interval bounded by the dijet system with $p_{\mathrm{T}}$ over veto threshold (e.g. $20 \mathrm{GeV}$ ). The fraction of events passing the veto is so-called gap fraction. The measured gap fraction and the values predicted by different generators are shown in fig. 4. The data are best described by HEJ [15], which is a parton-level event generator that provides an all-order description of wide-angle emissions of similar $p_{\mathrm{T}}$. The theoretical uncertainties are dominated by the choice of renormalization/factorization scale (single HEJ parameter). Fig. 4 shows that in most of the phase-space the experimental uncertainty is smaller than the theoretical one. These results can therefore be used to constrain the event generator modeling of QCD radiation between widely separated jets.

Less inclusive (and more challenging) class of jet measurements is a production of more than two jets in the final state [16]. Multijet events are very useful for testing higher orders or perturbative QCD. Unlike in previous analyses, the events are selected using multi (2 or 3 ) jet trigger. In order to provide precise check of NLO predictions (provided by NLOJet++), only jets with $p_{\mathrm{T}}>60$ $\mathrm{GeV}$ and $|y|<2.8$ were considered: those have the lowest JES uncertainty. The results were compared with LO and NLO generators. All models reproduce well the main features of the multijet 

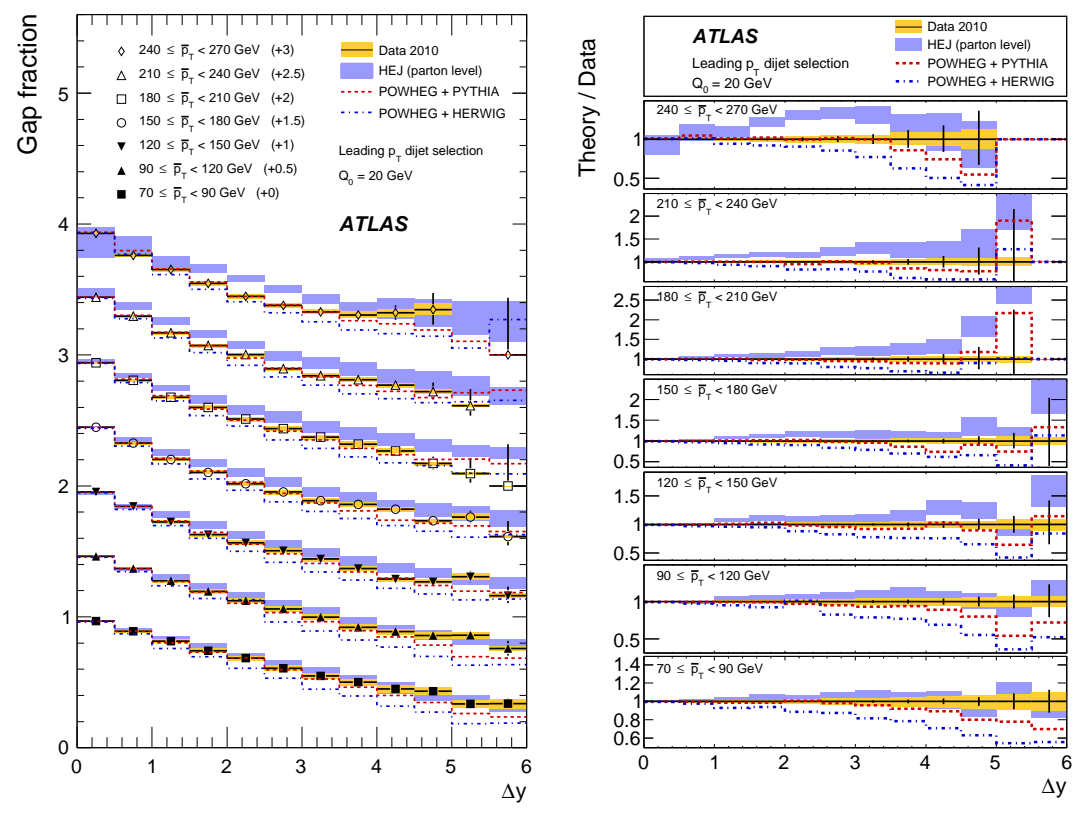

Figure 4: Gap fraction as a function of $\Delta y$ for various mean $p_{\mathrm{T}}$ slices. Data are black points with error bars representing statistical uncertainty and yellow band representing the total systematic uncertainty. The blue band is representation of theoretical uncertainty in HEJ calculation. The dashed lines represent POWHEG predictions with showering, hadronization and underlying event done by Pythia or HERWIG/JIMMY, respectively. The left plot shows measured values (multiplied by factors indicated in the legend) while the right plot shows ratios of theory predictions and the data. Figures are from [13].

data. Experimental and theoretical uncertainties are comparable, but as experimental uncertainty is expected to be reduced with larger data samples, future comparison of multijet measurements with NLO pQCD calculations will be useful for constraining PDFs and strong coupling constant value.

At the LHC, the electroweak-scale objects are often created as highly boosted. Thus if they decay hadronically, their decay products might be contained in a single jet. Substructure studies offer techniques to distinguish these single jets of interest from QCD background. However, most of those studies rely on the assumption that the internal jet structure is well modeled by MC and has not been tried with data.

ATLAS measured some of the relevant variables in the sample of QCD jets [17]. The focus is on anti- $k_{t}$ jets with $R=1.0$ and Cambridge-Aachen [18] jets with $R=1.2$. These large jets were split to smaller jets and filtered in order to retain particles relevant for the hard process and reduce the effect of underlying event and pile-up. The mass of individual jets encodes the information about the parton shower and potential presence of heavy particle decays. This analysis was the world's first measurement of the filtered jet mass and the result shows that Monte Carlo jet modeling is in a good agreement with data and that ATLAS is capable of delivering measurements requiring knowledge of jet substructure.

The production of prompt photon at hadron colliders is very useful for testing QCD. Photons provide colourless probe of quarks in the hard partonic interaction. Their production is directly sensitive to the gluon content of the proton, so the measurement of prompt photon cross-section 

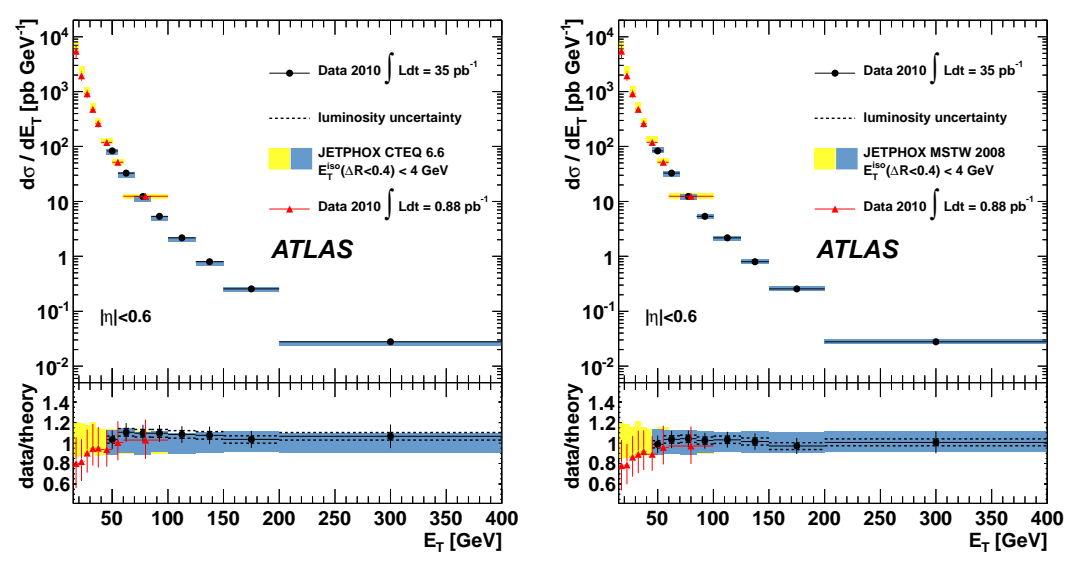

Figure 5: Measured and expected inclusive prompt photon differential cross-section versus photon $E_{\mathrm{T}}$ in a range $|\eta|<0.6$. The black dots are results for photons with $E_{\mathrm{T}}>45 \mathrm{GeV}$ obtained from full 2010 dataset, while red dots have been measured using only a fraction of data with low instantaneous luminosity. The theory NLO prediction and uncertainty is presented by yellow and blue bands. The left plot uses CTEQ 6.6 PDFs, while the right one uses MSTW08 PDFs. Figures are from [19].

can be used to constrain gluon PDFs. This measurement also improves our knowledge of QCD backgrounds in searches for Higgs boson or new physics phenomena.

In ATLAS, photons are identified as narrow, isolated showers in the electromagnetic calorimeter that cannot be matched to a track. Tracker information is also used to reject photons from conversions. The shower isolation is important to reject other neutral particles like $\pi^{0}$. The shape of the isolation energy is determined from data. The measured values of prompt photon cross-section [19] are shown in fig. 5. The data are compared to NLO QCD predictions (by JETPHOX [20]) with 2 different sets of PDFs to show the sensitivity. Data agree very well with the theory prediction with the exception of low $E_{\mathrm{T}}$ region where the fragmentation is high. The main source of experimental uncertainty is the EM energy scale.

\section{4. $W$ and $Z$ boson}

Electroweak interaction can be tested by studying the production of $\mathrm{W}$ and $\mathrm{Z}$ bosons. These heavy particles are also used as "standard candles" to calibrate our detector. The measurement of cross-sections of $\mathrm{W}$ and $\mathrm{Z}$ decaying to leptons [21 -23] was among the first measurement performed at ATLAS. The cross-section was measured in electron, muon and tau decay channels.

The measurement of $\mathrm{W}$ cross-section requires the presence of one well reconstructed lepton with $p_{\mathrm{T}}>20 \mathrm{GeV}$ (in the tau channel also the upper cut of $60 \mathrm{GeV}$ is applied) and sizable missing transverse energy $E_{\mathrm{T}}^{\text {miss }}>25 \mathrm{GeV}$ ( $30 \mathrm{GeV}$ in the tau channel). The events are selected using single lepton trigger in $e / \mu$ channels and with the tau and $E_{\mathrm{T}}^{\text {miss }}$ trigger in the tau channel.

In the $\mathrm{Z}$ decays, the presence of two well reconstructed leptons with opposite charge and invariant mass in a range $66<m_{l l}<116 \mathrm{GeV}$ is required in the electron and muon channels. In the $\tau \tau$ channel, the search is done in 4 sub-channels ( 2 semileptonic, $e \mu$ and $\mu \mu$ final states). The events in all channels are selected using single electron or single muon trigger. The results in all 


\begin{tabular}{llllll}
\hline \hline & $\sigma[\mathrm{nb}]$ & stat. & sys. & lumi. & acc. \\
\hline$\sigma(W \rightarrow e v)$ & 10.255 & 0.031 & 0.190 & 0.349 & 0.156 \\
$\sigma(W \rightarrow \mu v)$ & 10.210 & 0.030 & 0.166 & 0.347 & 0.153 \\
$\sigma(W \rightarrow \tau v)$ & 11.1 & 0.3 & 1.7 & 0.4 & \\
$\sigma\left(Z / \gamma^{*} \rightarrow e e\right)$ & 0.952 & 0.010 & 0.026 & 0.032 & 0.019 \\
$\sigma\left(Z / \gamma^{*} \rightarrow \mu \mu\right)$ & 0.935 & 0.009 & 0.009 & 0.032 & 0.019 \\
$\sigma\left(Z / \gamma^{*} \rightarrow \tau \tau\right)$ & 0.97 & 0.07 & 0.06 & 0.03 & \\
\hline \hline
\end{tabular}

Table 1: Results [21 - 23] of the inclusive $\mathrm{W}$ and $\mathrm{Z}$ cross-section measurements in lepton channels including the estimated statistical and systematical error as well as the luminosity and acceptance uncertainty. In the $\tau$ channels, the acceptance uncertainty is included in the systematic. The $Z \rightarrow \tau \tau$ result is a combination of 4 sub-channels.

studied channels of $\mathrm{W}$ and $\mathrm{Z}$ decays are compatible with NNLO predictions [21] from FEWZ program $(\sigma(W \rightarrow \ell v)=10.46 \pm 0.52 \mathrm{nb}$ and $\sigma(Z \rightarrow \ell \ell)=0.96 \pm 0.05 \mathrm{nb})$, and precision in electron and muon channels is comparable with the theoretical uncertainty.

The main systematics in electron and muon channels comes from the luminosity uncertainty (3.4\% [24]). The systematics in the tau channels with hadronic tau decays is dominated by the uncertainty on the tau efficiency and energy scale (10\% and $8 \%$, respectively), while the uncertainty on the leptonic tau decays is mainly statistical.

In both $\mathrm{W}$ and $\mathrm{Z}$ cross-section measurements the electroweak background has been estimated from MC simulation and QCD background from the data. The result is summarized in tab. 1.

Very important prediction of the Standard Model is the ratio of $\mathrm{W}$ and $\mathrm{Z}$ cross-sections as well as ratio of branching ratios of their decay to different lepton flavours. The ratio of $\mathrm{W}$ and $\mathrm{Z}$ crosssections measured within kinematic and geometrical acceptance of the ATLAS detector (fiducial cross-section) is shown in fig. 6. It is compared to the theory predictions that were evaluated within the ATLAS fiducial volume using FEWZ and DYNNLO programs. Fig. 6 also shows the ratio of branching ratios of $\mathrm{Z}$ and $\mathrm{W}$ compared to the world average and the SM prediction.

Measurement of the cross-section of events where $\mathrm{W}$ boson and jets are produced simultaneously is very important test of the pQCD [25]. Also, events of this type form important background for the Higgs or SUSY searches. In this measurement, only the jets in the rapidity range $|y|<2.8$ and well separated from the lepton from $\mathrm{W}$ decay $(\Delta R>0.5)$ are considered. The jets produced in pile-up collisions are removed using Jet Vertex Fraction (JVF): sum of momenta coming from different than primary vertex. The dominant uncertainty sources in this analysis are JES (10\%) and pile-up jets removal uncertainty (up to $7 \%$ at low $p_{\mathrm{T}}$ ). The results presented in fig. 7 are in a good agreement with predictions of the multi-parton matrix element generators (ALPGEN [26] and SHERPA [27]) and NLO matrix elements calculators MCFM [28] and BLACKHAT-SHERPA [29]. The Pythia predictions fail to describe events with more than one additional jet.

Diboson production is testing gauge boson self-interaction and thus non-abelian nature of the electroweak interaction. Possible physics beyond the Standard Model can demonstrate itself by anomalous values of the triple gauge coupling between $\mathrm{W}$ and $\mathrm{Z}$ and/or by presence of neutral trilinear gauge coupling (ZZZ and $\gamma Z Z$ ) that is explicitly forbidden in the Standard Model. ATLAS 

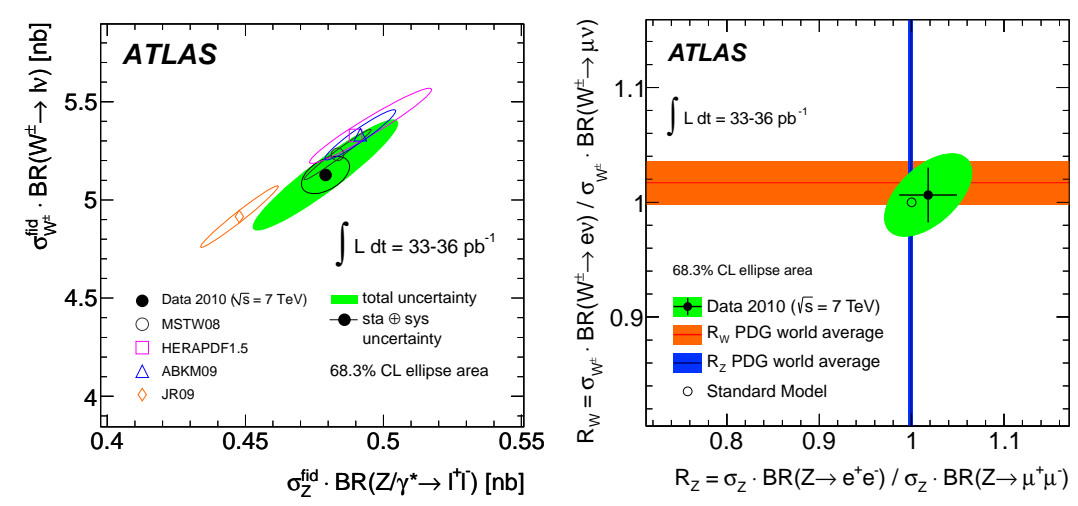

Figure 6: The left plot shows measured and predicted fiducial cross sections times leptonic branching ratios, $(\sigma(W+)+\sigma(W-))$ vs. $\sigma\left(Z / \gamma^{*}\right)$. The ellipses illustrate the $68 \%$ confidence level (CL) coverage for total uncertainties (full green) and excluding the luminosity uncertainty (open black). The theory prediction are evaluated within the ATLAS fiducial volume. The uncertainties of the theoretical predictions correspond to the PDF uncertainties only. The right plot presents the correlated measurement of the $e$-to- $\mu$ cross section ratios in the $\mathrm{W}$ and the $\mathrm{Z}$ channels. The vertical (horizontal) band represents the uncertainty of the corresponding $\mathrm{Z}(\mathrm{W})$ branching fractions based on the current world average data. The green ellipse illustrates the $68 \% \mathrm{CL}$ for the correlated measurement of $R_{W}$ and $R_{Z}$, while the error bars correspond to the one-dimensional uncertainties of either $R_{W}$ or $R_{Z}$, respectively. Figures are from [21].
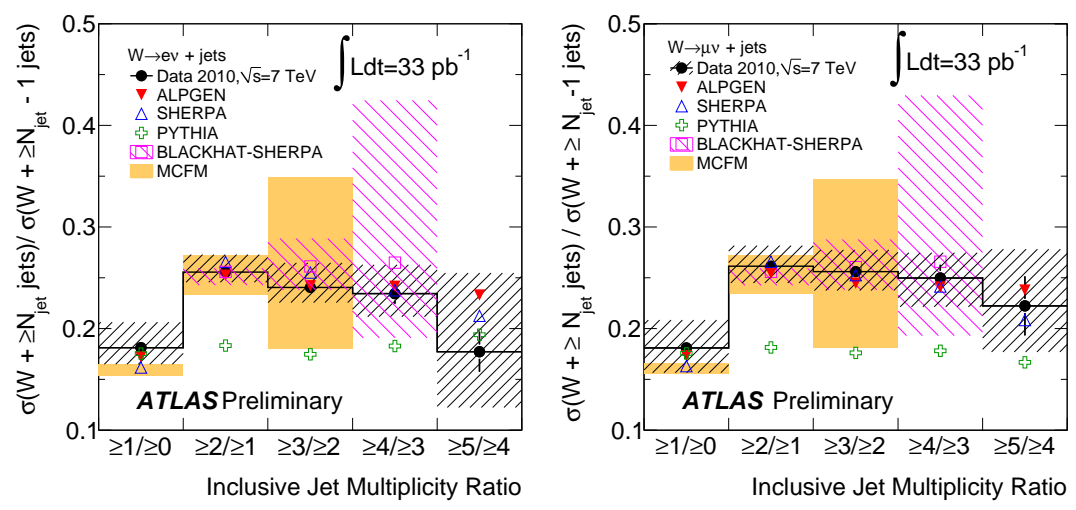

Figure 7: W+jets cross section ratio as a function of corrected jet multiplicity. For the data, the statistical uncertainties are shown by the vertical bars, and the combined statistical and systematic uncertainties are shown by the black-hashed regions. Also shown are MC predictions. The theoretical uncertainties are shown only for MCFM (NLO prediction for $N_{\text {jet }}<2$ and a LO prediction for $N_{\text {jet }}=3$ ) and BLACKHATSHERPA (NLO prediction for $N_{\text {jet }}<3$ and a LO prediction for $N_{\text {jet }}=4$ ). The left plot is for electron channel and right for the muon channel. Figures are from [25]. 


\begin{tabular}{lll}
\hline \hline & measured $\sigma$ [pb] & SM expected $\sigma$ [pb] \\
\hline$\sigma_{\text {tot }}(W W)$ & $51.0 \pm 4.5$ (stat.) \pm 6.4 (syst.) \pm 1.9 (lumi.) & $46 \pm 3$ \\
$\sigma_{\text {tot }}(W Z)$ & $20.5_{-2.8}^{+3.1}$ (stat. $)_{-1.3}^{+1.4}(\text { syst. })_{-0.8}^{+0.9}($ lumi.) & $17.3_{-0.8}^{+1.3}$ \\
$\sigma_{\text {tot }}(Z Z)$ & $8.5_{-2.3}^{+2.7}$ (stat. $)_{-0.3}^{+0.4}$ (syst.) \pm 0.3 (lumi.) & $6.5_{-0.2}^{+0.3}$ \\
\hline \hline
\end{tabular}

Table 2: Results [30 - 32] of the total diboson cross-section measurements in lepton channels including the estimated statistical and systematical error as well as the luminosity uncertainty. The central column shows measured values, while the rightmost presents Standard Model NLO prediction.
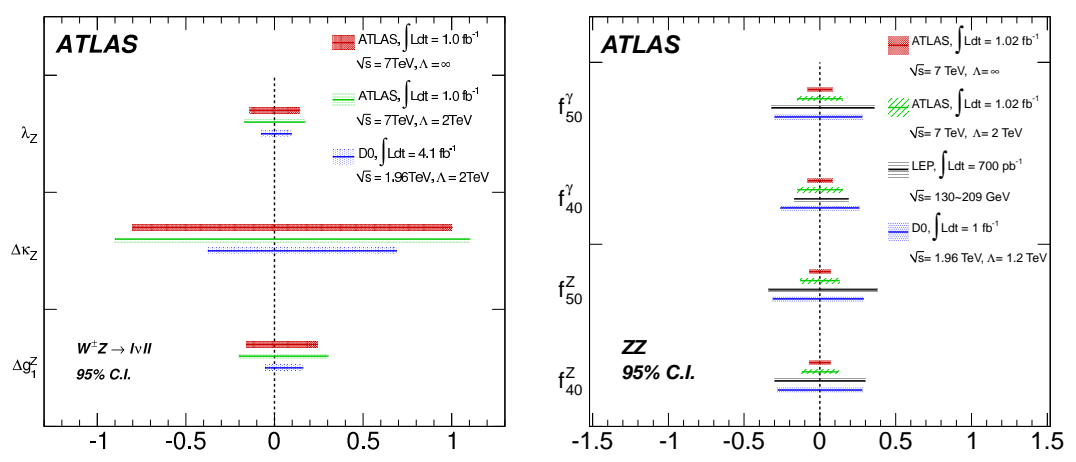

Figure 8: Left plot shows $95 \%$ confidence intervals for anomalous couplings from ATLAS and D0 experiments. For ATLAS, limits are shown for 2 different cut-off scales $\Lambda$. The right plot presents anomalous neutral triple gauge coupling (nTGC) 95\% confidence intervals from ATLAS, LEP and Tevatron experiments. The value expected in the Standard Model is zero in both plots. Figures are from [31,32].

measurement of WW [30], WZ [31] and ZZ [32] production did not find any deviations from NLO Standard Model predictions provided by MC@NLO (WW,WZ) and MCFM (ZZ) programs. All 3 measurements are exploiting electron and muon channels and events are in all cases selected with the single lepton trigger. The measured and expected cross-sections are presented in tab. 2 .

Finally, ATLAS also evaluated limits on the anomalous gauge couplings. This is shown in fig. 8 and compared with the results obtained from Tevatron and LEP experiments. The ATLAS limits are comparable or better than the ones from previous experiments. Because the uncertainty in $\mathrm{WZ}$ and $\mathrm{ZZ}$ production is dominated by statistical error, it is expected that these limits will be improved when whole 2011 dataset will be fully analyzed.

\section{Top Quark}

The top quark is the most massive known fundamental constituent of nature and the only one with a mass of the same order of magnitude as the electroweak symmetry scale. The study of top quark production and decay therefore allows for a precision tests of the Standard Model and to constrain the nature of the electroweak spontaneous symmetry breaking, in particular the mass range of the Higgs boson. The top quark production is also important background in many searches for the Higgs boson as well as for signs of a new physics, such as supersymmetry. Finally, due to its 
large mass, the top quark is extremely short lived and decays to $\mathrm{W}$ and $b$-quark before it hadronizes, thus allowing for direct study of its properties.

At the LHC, the top quarks are predominantly produced in $t \bar{t}$ pairs. At $\sqrt{s}=7 \mathrm{TeV} p p$ collisions, the production cross-section is dominated by the gluon fusion over quark annihilation and its value to NNLO, assuming $m_{\text {top }}=172.5 \mathrm{GeV}$, is $165_{-16}^{+11} \mathrm{pb}$ [33]. The $\mathrm{W}$ boson produced by a top quark can decay either to leptons (electron, muon or tau decaying to leptons) or jets (light quark jets or hadronic tau decays) and therefore the $t \bar{t}$ final state is very varied: there are $b$-jets, high $p_{\mathrm{T}}$ jets and/or high $p_{\mathrm{T}}$ leptons from $\mathrm{W}$ decays and large $E_{\mathrm{T}}^{\text {miss }}$ due to neutrino in W leptonic decays.

The $t \bar{t}$ decays are classified as dileptonic (both W's decay to leptons), semi-leptonic (one W decay to leptons) or fully hadronic otherwise. ATLAS has measured $\sigma_{t \bar{t}}$ in dileptonic [34] as well as semi-leptonic $t \bar{t}$ decays $[35,36]^{4}$. The main Standard Model background for $t \bar{t}$ events are W/Z production associated with jets and QCD multi-jet events.

The events in the dilepton channel are selected using single lepton trigger. Afterwards, presence of two well reconstructed isolated leptons with $p_{\mathrm{T}}>25 \mathrm{GeV}$ is required. Additionally, significant amount of $E_{\mathrm{T}}^{\mathrm{miss}}$ is required in order to suppress the multi-jet background. Finally, a parallel selection requires that at least one of the jets is identified as $b$-quark candidate (" $b$ tagged") by an algorithm that uses the characteristics of $b$-hadrons (e.g. observable flight distance from the primary vertex) to separate $b$-jets from other types of jets. Usage of $b$-tagging results in higher signal purity, but the price paid is more difficult assessment of the systematical uncertainty. Therefore in the statistical combination [38], only the dileptonic results obtained without $b$-tagging are included as measurement using $b$-tagging was not finalized at that time. The cross-section measured in the dileptonic channel without $b$-tagging is $\sigma_{t \bar{t}}=177 \pm 6$ (stat.) $)_{-14}^{+17}$ (sys.) \pm 8 (lumi.) pb. The measurement requiring at least 1 jet consistent with the $b$-quark hypothesis yields $\sigma_{t \bar{t}}=183 \pm 6$ (stat. $)_{-14}^{+18}$ (sys. $)_{-7}^{+8}$ (lumi.) pb.

The signature of $t \bar{t}$ semileptonic decays is presence of a high- $p_{\mathrm{T}}$ lepton from the $\mathrm{W}$ decay and several jets. The events for this analysis are again selected with single lepton triggers and are required to have isolated, well reconstructed lepton and $E_{\mathrm{T}}^{\text {miss }}$ value consistent with W leptonic decay. In 2011 data, the measurement is done in 6 sub-channels, depending on the jet multiplicity ( 3,4 or 5 and more) and lepton flavour $(e$ or $\mu)$. Unlike 2010 lepton+jets analysis, the search [35] does not rely on $b$-tagging. The measured cross-section is $\sigma_{t \bar{t}}=179.0 \pm$ 3.9 (stat.) \pm 9.0 (sys.) \pm 6.6 (lumi.) pb.

The $t \bar{t}$ cross-section was also measured in the events where one $\mathrm{W}$ decays to $\mu$ and the other to a $\tau$ lepton that decays hadronically. Single muon trigger was used to select events for this analysis. All selected events are required to have exactly one isolated muon and no identified electrons. The $\tau$ candidates are selected from reconstructed jets using Boosted Decision Trees (BDT) [39]. In addition, at least one $b$-tagged jet and $E_{\mathrm{T}}^{\text {miss }}>30 \mathrm{GeV}$ is required. The resulting cross-section is $\sigma_{t \bar{t}}=142 \pm 21$ (stat.) $)_{-16}^{+20}$ (syst.) \pm 5 (lumi.) pb.

All cross-sections measured in various $t \bar{t}$ decays are consistent with NNLO theoretical prediction. The statistical combination [38] used 2011 dilepton results without b-tagging and 2010 lepton+jets results with $b$-tagging to obtain value $\sigma_{t \bar{t}}=176 \pm 5$ (stat.) ${ }_{-10}^{+13}$ (syst.) \pm 7 (lumi.) pb. These

\footnotetext{
${ }^{4}$ This was true at the time of the conference. Afterwards, ATLAS has also published results in fully hadronic channel [37].
} 

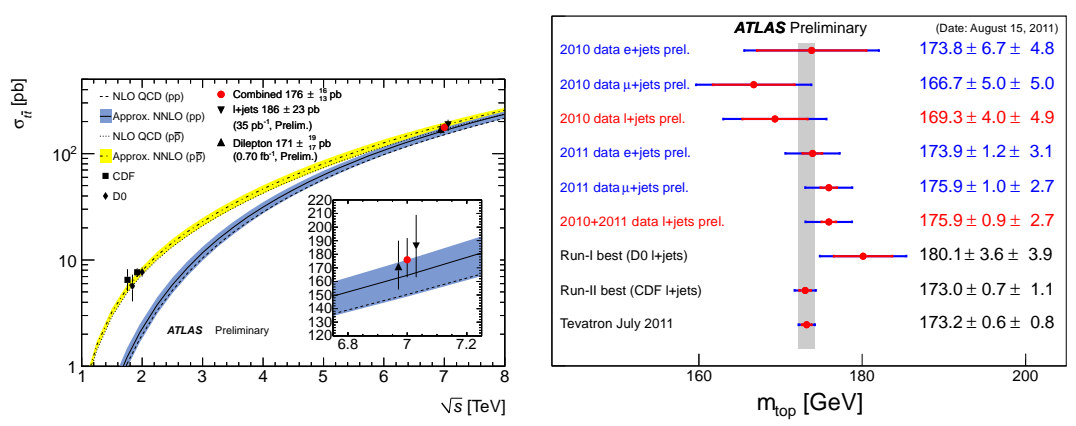

Figure 9: Left plot shows dependence of $\sigma_{t \bar{t}}$ on $\sqrt{s}$ predicted for $p p$ and $p \bar{p}$ collisions, assuming $m_{\mathrm{top}}=$ 172.5 GeV. ATLAS results in dilepton and single lepton channels as well as their combination are presented together with Tevatron results. The total uncertainty of experimental results is shown as vertical bar. The measurements at the same $\sqrt{s}$ are offset for the sake of clarity. Figure is from [38]. The right plot presents the results on $m_{\text {top }}$ from the individual analyses and their combination, compared to selected results from other measurements and present average value from the Tevatron experiments. Figure is from [40].

results are compared to previous measurements and theory prediction in fig. 9.

Based on the 2011 data, the mass of the top quark has been measured using the template method in the lepton+jets channels [40]. The events are recorded with single lepton (electron or muon) trigger and are required to have exactly one well reconstructed and isolated lepton with $p_{\mathrm{T}}>$ 25 (20) $\mathrm{GeV}$ for electrons (muons). Additionally, at least 4 reconstructed jets with $p_{\mathrm{T}}>25 \mathrm{GeV}$ (at least one of them $b$-tagged) are required and sizable $E_{\mathrm{T}}^{\text {miss }}$ compatible with leptonic $\mathrm{W}$ decay is demanded as well. In this two-dimensional template analysis, both $m_{\text {top }}$ and a global jet energy scale factor (JSF) are simultaneously determined from the distributions of reconstructed top and W masses. The global JSF correction is obtained mostly from observed differences between predicted and measured distributions of $\mathrm{W}$ invariant masses. In this method, the systematic uncertainty on $m_{\text {top }}$ stemming from JES uncertainty is reduced and partly transformed to an additional statistical uncertainty on $m_{\text {top }}$ due to $2 \mathrm{D}$ fit. The combination of results electron and muon channels with the results from 2010 yields $m_{\text {top }}=175.9 \pm 0.9$ (stat.) \pm 2.7 (syst.) $\mathrm{GeV}$. The mass estimates in all channels and comparison to Tevatron results are presented in fig. 9.

A single top quark can be produced via electroweak interaction either in $\mathrm{W}$-mediated $t$ - and $s$ - channels or in the $W t$ channel, where virtual $b$-quark mediates the associated production of $\mathrm{W}$ boson and a top quark. Using cut-based analysis and neural network, ATLAS was able to measure cross-section of a single top production in the $t$-channel [41]. The result $\sigma_{t}=90_{-22}^{+32} \mathrm{pb}$ is consistent with the SM NNLO expectation value of $\sigma_{t}=64.6_{-2.6}^{+3.3} \mathrm{pb}$ [42] within 1.1 standard deviations.

The analyses looking for single top-quark production in $s$ [43] and $\mathrm{W} t$ channels [44] using 0.7 $\mathrm{fb}^{-1}$ were not sensitive enough to measure the cross-section: an upper limit on single top production was set instead. The observed $95 \%$ confidence limit on $\mathrm{W} t$ production is $39 \mathrm{pb}$ (cross-section expected by the SM is $15.7 \mathrm{pb}$ [42]) and $95 \% \mathrm{CL}$ on the $s$-channel single top quark production is $26.5 \mathrm{pb}$ (cross-section expected by the SM is $4.6 \mathrm{pb}[42]$ ).

\section{Conclusion}

Large Hadron Collider has performed excellently in 2010 and 2011 and ATLAS detector was 
able to record more than $5 \mathrm{fb}^{-1}$ of good data. This contribution presents overview of a fraction of results from ATLAS analyses focusing on various aspects of the Standard Model using 2010 data and up to $1 \mathrm{fb}^{-1}$ of 2011 data. All known particles and vertices of the SM have been successfully rediscovered and sensitivity in some of the measurements is large enough to deliver the world-best results. All results are consistent with the (N)NLO Standard Model predictions within uncertainties. The complete list of ATLAS results related to the Standard Model is available online [45].

\section{References}

[1] The ATLAS Collaboration, The ATLAS Experiment at the CERN Large Hadron Collider, JINST 3 (2008) S08003.

[2] L. Evans, (ed.) and P. Bryant, (ed.), LHC Machine, JINST 3 (2008) S08001.

[3] The ATLAS Collaboration, Charged-particle multiplicities in pp interactions measured with the ATLAS detector at the LHC, New J. Phys. 13 (2011) 053033.

[4] T. Sjostrand, S. Mrenna and P. Skands, PYTHIA 6.4 physics and manual, JHEP 05 (2006) 026, [arXiv:hep-ph/0603175].

[5] The ATLAS Collaboration, Measurement of the Inelastic Proton-Proton Cross-Section at $\sqrt{s}=7 \mathrm{TeV}$ with the ATLAS Detector, Nat. Commun. 2 (2011) 463 [arXiv: 1104 . 0326].

[6] The ATLAS Collaboration, Expected Performance of the ATLAS Experiment - Detector, Trigger and Physics, [arXiv:0901.0512].

[7] M. Cacciari, G. P. Salam and G. Soyez, The Anti- $k_{t}$ jet clustering algorithm, JHEP 0804, 063 (2008) [arXiv:0802.1189].

[8] The ATLAS Collaboration, Jet energy scale and its systematic uncertainty in proton-proton collisions at $\sqrt{s}=7 \mathrm{TeV}$ in ATLAS 2010 data, ATLAS-CONF-2010-032, https://cdsweb.cern.ch/record/1337782.

[9] The ATLAS Collaboration, Measurement of inclusive jet and dijet cross sections in proton-proton collision data at $7 \mathrm{TeV}$ centre-of-mass energy using the ATLAS detector, ATLAS-CONF-2010-047, https://cdsweb.cern.ch/record/1338578.

[10] Z. Nagy, Next-to-leading order calculation of three jet observables in hadron hadron collision, Phys. Rev. D68 (2003) 094002, [arXiv: hep-ph / 0307268 ].

[11] J. Pumplin et al., New generation of parton distributions with uncertainties from global QCD analysis, JHEP 07 (2002) 012, [arXiv: hep-ph/ 0201195].

[12] The ATLAS Collaboration, Measurement of Dijet Azimuthal Decorrelations in pp Collisions at $\sqrt{s}=7 \mathrm{TeV}$, Phys. Rev. Lett. 106 (2011) 172002 [arXiv: 1102 . 2696].

[13] The ATLAS Collaboration,15.7 Measurement of dijet production with a veto on additional central jet activity in pp collisions at $\sqrt{s}=7 \mathrm{TeV}$ using the ATLAS detector, JHEP 09 (2011) 053 [arXiv:1107.1641].

[14] I. I. Balitsky and L. N. Lipatov, The Pomeranchuk Singularity in Quantum Chromodynamics, Sov. J. Nucl. Phys. 28 (1978) 822-829.

[15] J. R. Andersen and J. M. Smillie, High Energy Description of Processes with Multiple Hard Jets, Nucl. Phys. Proc. Suppl. 205-206 (2010) 205-210, [arXiv: 1007 . 444 9]. 
[16] The ATLAS Collaboration, Measurement of multi-jet cross sections in proton-proton collisions at a 7 TeV center-of-mass energy, Eur.Phys.J. C 71 (2011) 1763 [arXiv: 1107.2092 ].

[17] The ATLAS Collaboration, Measurement of Jet Mass and Substructure for Inclusive Jets in $\sqrt{s}=7 \mathrm{TeV}$ pp Collisions with the ATLAS Experiment, ATLAS-CONF-2011-073, https://cdsweb.cern.ch/record/1352454.

[18] Y. L. Dokshitzer, G. D. Leder, S. Moretti, and B. R. Webber, Better Jet Clustering Algorithms, JHEP 08 (1997) 001, [arXiv : hep-ph/9707323].

[19] The ATLAS Collaboration, Measurement of the inclusive isolated prompt photon cross-section in pp collisions at $\sqrt{s}=7 \mathrm{TeV}$ using $35 \mathrm{pb}^{-1}$ of ATLAS data, Phys.Lett.B 706 (2011) 150-167 [arXiv: 1108.0253$]$.

[20] S. Catani, M. Fontannaz, J. P. Guillet and E. Pilon, Cross-section of isolated prompt photons in hadron hadron collisions, JHEP 0205, 028 (2002) [hep-ph/ 0204023 ].

[21] The ATLAS Collaboration, Measurement of the inclusive $W^{ \pm}$and $Z / \gamma$ cross sections in the electron and muon decay channels in pp collisions at $\sqrt{s}=7 \mathrm{TeV}$ with the ATLAS detector, CERN-PH-EP-2011-143 [arXiv:1109.5141].

[22] The ATLAS Collaboration, Measurement of the W to $\tau \nu$ Cross Section in pp Collisions at $\sqrt{s}=7 \mathrm{TeV}$ with the ATLAS experiment, CERN-PH-EP-2011-122 [arXiv: 1108 . 4101].

[23] The ATLAS Collaboration, Measurement of the $Z$ to $\tau \tau$ Cross Section with the ATLAS Detector, CERN-PH-EP-2011-097 [arXiv:1108.2016].

[24] The ATLAS Collaboration, Updated Luminosity Determination in pp Collisions at $\sqrt{s}=7$ TeV using the ATLAS Detector, ATLAS-CONF-2011-011, https://cdsweb.cern.ch/record/1334563.

[25] The ATLAS Collaboration, Measurement of the production cross section for $W$-bosons in association with jets in pp collisions using $33 \mathrm{pb}^{-1}$ at $\sqrt{\mathrm{s}}=7 \mathrm{TeV}$ with the ATLAS detector, ATLAS-CONF-2011-060, https://cdsweb.cern.ch/record/1344778.

[26] M. L. Mangano, M. Moretti, F. Piccinini, R. Pittau and A. D. Polosa, ALPGEN, a generator for hard multiparton processes in hadronic collisions, JHEP 0307, 001 (2003) [hep-ph/ 0206293 ].

[27] T. Gleisberg, S. Hoeche, F. Krauss, M. Schonherr, S. Schumann, F. Siegert and J. Winter, Event generation with SHERPA 1.1, JHEP 0902, 007 (2009) [arXiv: 0811 . 4622].

[28] J. M. Campbell, R. K. Ellis, and D. L. Rainwater, Next-to-leading order QCD predictions for W+2jet and Z+2jet production at the CERN LHC, Phys. Rev. D68 (2003) 094021.

[29] C. F. Berger et al., Precise predictions for $W+4$ jet production at the Large Hadron Collider, [arXiv:1009.2338].

[30] The ATLAS Collaboration, Measurement of the $W^{+} W^{-}$production cross section in proton-proton collisions at $\sqrt{s}=7 \mathrm{TeV}$ with the ATLAS detector, ATLAS-CONF-2011-110, https://cdsweb.cern.ch/record/1373412.

[31] The ATLAS Collaboration, Measurement of the WZ production cross section and limits on anomalous triple gauge couplings in proton-proton collisions at $\sqrt{s}=7 \mathrm{TeV}$ with the ATLAS detector, CERN-PH-EP-2011-184, [arXiv:1111.5570].

[32] The ATLAS Collaboration, Measurement of the ZZ production cross section and limits on anomalous neutral triple gauge couplings in proton-proton collisions at $\sqrt{s}=7 \mathrm{TeV}$ with the ATLAS detector, CERN-PH-EP-2011-166, [arXiv:1110.5016]. 
[33] M. Beneke, P. Falgari, S. Klein and C. Schwinn, Hadronic top-quark pair production with NNLL threshold resummation, Nucl. Phys. B 855, 695 (2012) [arXiv: 1109.1536 ].

[34] The ATLAS Collaboration, Measurement of the top quark pair production cross section in pp collisions at $\sqrt{s}=7 \mathrm{TeV}$ in dilepton final states with ATLAS, ATLAS-CONF-2011-100, https://cdsweb.cern.ch/record/1369215.

[35] The ATLAS Collaboration, Measurement of the t $\bar{t}$ production cross-section in pp collisions at $\sqrt{s}=7$ TeV using kinematic information of lepton+jets events, ATLAS-CONF-2011-121, https://cdsweb.cern.ch/record/1376413.

[36] The ATLAS Collaboration, Measurement of the top quark pair production cross section in pp collisions at $\sqrt{s}=7 \mathrm{TeV}$ in $\mu+\tau$ final states with ATLAS, ATLAS-CONF-2011-119, https://cdsweb.cern.ch/record/1376411.

[37] The ATLAS Collaboration, Measurement of $t \bar{t}$ production in the all-hadronic channel in $1.02 \mathrm{fb}^{-1}$ of pp collisions at $\sqrt{s}=7 \mathrm{TeV}$ with the ATLAS detector, ATLAS-CONF-2011-140, https://cdsweb.cern.ch/record/1385033.

[38] The ATLAS Collaboration, Measurement of the top quark pair production cross-section based on a statistical combination of measurements of dilepton and single-lepton final states at $\sqrt{s}=7$ TeV with the ATLAS detector, ATLAS-CONF-2011-108, https://cdsweb.cern.ch/record/1373410.

[39] The ATLAS Collaboration, Measurement of the Mis-identification Probability of $\tau$ Leptons from Hadronic Jets and from Electrons, ATLAS-CONF-2011-113, https://cdsweb.cern.ch/record/1375550.

[40] The ATLAS Collaboration, Measurement of the top quark mass from 2011 ATLAS data using the template method, ATLAS-CONF-2011-120, https://cdsweb.cern.ch/record/1376412.

[41] The ATLAS Collaboration, Measurement of the t-channel Single Top-Quark Production Cross Section in $0.70 \mathrm{fb}^{-1}$ of pp Collisions at $\sqrt{\mathrm{s}}=7 \mathrm{TeV}$ with the ATLAS detector, ATLAS-CONF-2011-101, https://cdsweb.cern.ch/record/1369217.

[42] N. Kidonakis, Next-to-next-to-leading-order collinear and soft gluon corrections for t-channel single top quark production, Phys. Rev. D 83 (2011) 091503 [arXiv: 1103.2792].

N. Kidonakis, Two-loop soft anomalous dimensions for single top quark associated production with a $W$ - or H-, Phys. Rev. D 82, 054018 (2010) [arXiv : 1005 . 4451 ].

N. Kidonakis, NNLL resummation for s-channel single top quark production, Phys. Rev. D 81, 054028 (2010) [arXiv: 1001.5034 ].

[43] The ATLAS Collaboration, Search for s-Channel Single Top-Quark Production in pp Collisions at $\sqrt{s}=7 \mathrm{TeV}$, ATLAS-CONF-2011-118, https://cdsweb.cern.ch/record/1376410.

[44] The ATLAS Collaboration, Search for $W+t$ single-top events in the dileptonic channel at ATLAS, ATLAS-CONF-2011-104, https://cdsweb.cern.ch/record/1369829.

[45] The ATLAS Collaboration, Standard Model Results, https://twiki.cern.ch/twiki/bin/view/AtlasPublic/StandardModelPublicResults. The ATLAS Collaboration, Top Public Results, https://twiki.cern.ch/twiki/bin/view/AtlasPublic/TopPublicResults. 